\title{
Preface to the special issue on intergranular and interphase boundaries in materials
}

\author{
Yuichi Ikuhara $\cdot$ Masanori Kohyama
}

Received: 8 March 2011 / Accepted: 8 March 2011/Published online: 22 March 2011

(C) Springer Science+Business Media, LLC 2011

The 13th International Conference on Intergranular and Interphase Boundaries in Materials (iib2010) was held at Shima Kanko Hotel, in Shima city, Mie prefecture, Japan, on June 27-July 2, 2010. This conference is held every 3 years, and serves as an unique venue for specialists and researchers in different areas of interface science to discuss and exchange their ideas with one another.

Many properties of materials are closely related to the structure of the interfaces and grain boundaries, in which vacancies, interstitials, dopants, segregated atoms, dislocations, and dangling bonds often accumulate. Recent progress in microstructural characterization, such as advanced electron microscopy, spectroscopic techniques, and new X-ray and nuclear methods, have made it possible to study interfaces and grain boundaries quantitatively, from the atomic scale through to the macroscopic scale. At the same time, theoretical calculations play a great role in these fields. We therefore find ourselves entering a new stage of the study of interfaces and grain boundaries. Quantitative information obtained in this new era of research will be used for the rational design and development of new materials.

Following the successful discussions in the past twelve iib conferences, various issues relating to interfacial phenomena in solids were chosen for particular coverage in

\section{Y. Ikuhara $(\varangle)$}

Institute of Engineering Innovation, The University of Tokyo,

Yayoi, Bunkyo, Tokyo 113-8656, Japan

e-mail: ikuhara@sigma.t.u-tokyo.ac.jp

\section{Kohyama}

Research Institute for Ubiquitous Energy Devices, National Institute of Advanced Industrial Science and Technology,

Midorigaoka, Ikeda, Osaka 563-8577, Japan

e-mail: m-kohyama@aist.go.jp iib2010. This conference focused on the atomic-scale characterization and properties of interfaces, including atomic level modeling, microscopy, spectroscopy, thermodynamics, mechanical, electrical, and magnetic properties. The iib conference series is devoted to the exposition of recent advances and outlining of future directions in the field of solid-solid interface science, and the following topics were selected as special sessions at iib2010.

1. Structural characterization of interfaces I: atomic structure

2. Structural characterization of interfaces II: electronic structure

3. Theoretical modeling of interfaces and related phenomena

4. Grain boundary engineering in metals and ceramics

5. Phase transformation and thermodynamics of interfaces

6. Segregation, diffusion, migration, and high temperature phenomena of interfaces

7. Surface properties and wetting

8. Interfacial phenomena in energy materials and devices

9. History of interface studies: past and future

In particular, the session "history of interface studies" was planned to review past interface research and present visions for the future. This session was also very useful for providing a deep perspective for young scientists who have recently entered this field.

Aiming for highly productive and positive outcomes of this conference, we arranged the best available venue for the conference sessions. Shima Kanko Hotel is one of Japan's most prestigious resort hotels, and provided all the delegates with ideal session rooms and accommodations. It also overlooks the spectacular Ago Bay, world-famous for 


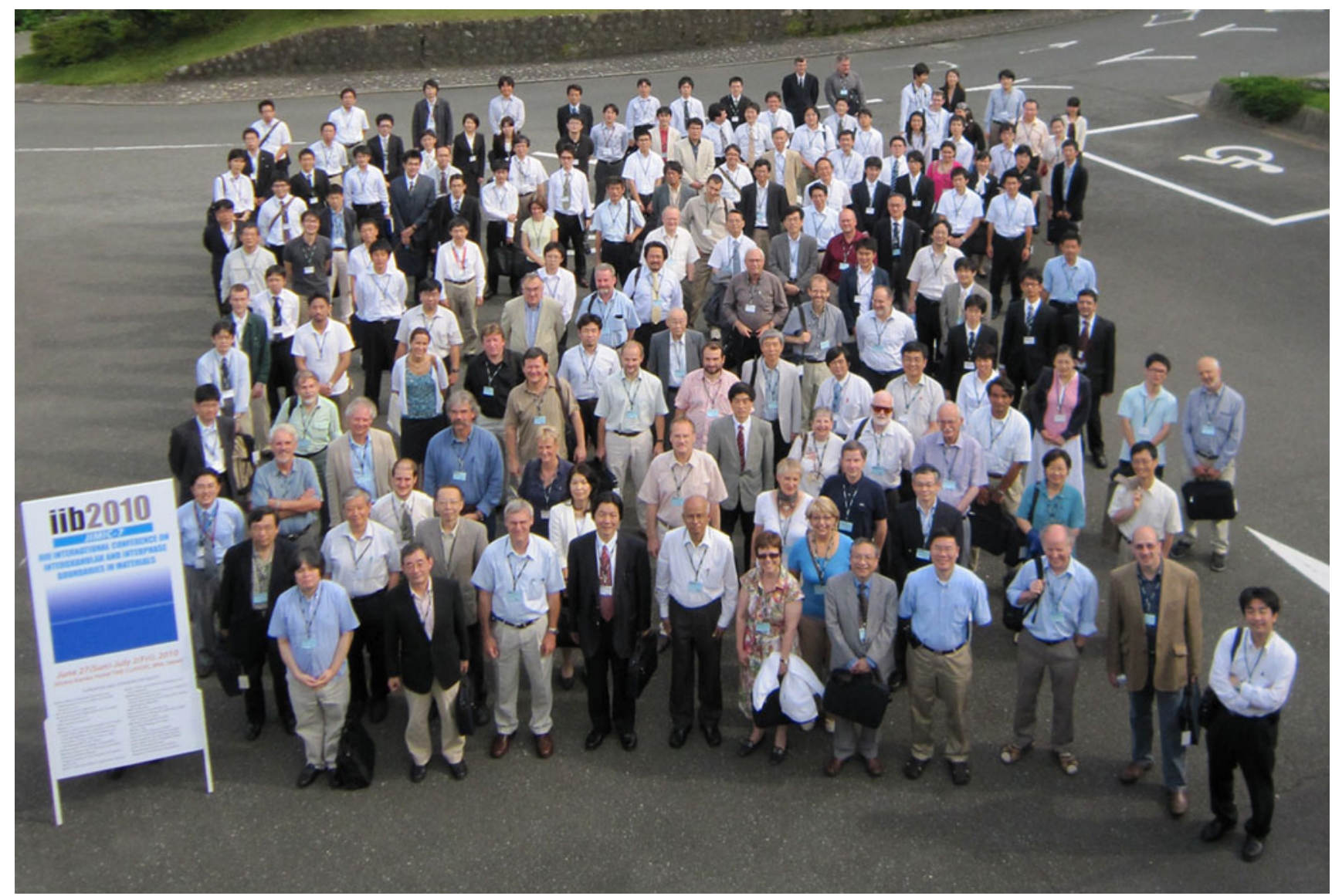

the beauty of the sea and for the rafts used for pearl cultivation, which was invented by the renowned Pearl King, Kokichi Mikimoto, back in 1893.

This issue contains 42 selected papers presented at the conference. The conference received over 170 abstracts, making it the largest symposium in the field of interface science. The papers collected in this issue were reviewed according to the strict reviewing policies of the journal and only those meeting the quality required for regular papers were accepted for publication.

The conference was organized by the iib2010 organizing committee members and the international advisory board, and co-organized as JIMIC 7 by Japan Institute of Metals. The main contributing organizations were the University of Tokyo, Grant-in-aid for Scientific Research on Priority Areas "Nano Materials Science for Atomic Scale Modification 474" from "Ministry of Education, Culture, Sports, Science and Technology (MEXT) of Japan" and "the Nanostructure Research Laboratory, Japan Fine Ceramics Center". The sponsors include Chubu Economic Federation, Japan Society for the Promotion of Science, The Ceramic Society of Japan, The Japanese Society of
Microscopy, The Iron and Steel Institute of Japan, The Japan Society of Applied Physics, JSPS 124th committee on advanced ceramics, JSPS 147th committee for Amorphous and Nano Materials, Mie Prefecture, Shima City, The Murata Science Foundation, Research Foundation for the Electrotechnology of Chubu, Iketani Science and Technology Foundation, Asian Office of Aerospace Research and Development, Air Force Office of Scientific Research, USA, JEOL Ltd., Hitachi High-Technologies Corporation, Topcon Technohouse Corporation, FEI company Japan Ltd., Gatan Inc., Nanofactory Instruments AB, and Merging Atomistic and Continuum Analysis of Nanometer Length-Scale Metal-Oxide Systems for Energy and Catalysis Applications (MACAN).

We hope this special issue contributes a lot to further enhance research in the field of materials interfaces. We thankfully acknowledge the great efforts of the iib2010 organizing committee members and the international advisory board members, particularly Professor Naoya Shibata as the general secretary and Professor Wayne Kaplan for intensively supporting to edit this JMS special issue. 